Volume 8, No. 7, July - August 2017

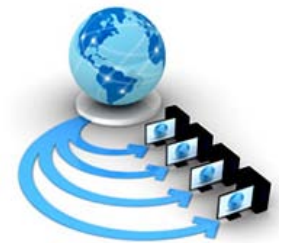

International Journal of Advanced Research in Computer Science

RESEARCH PAPER

Available Online at www.ijarcs.info

\title{
MATLAB BASED (GRAPHICAL USER INTERFACE) ENVIRONMENT SENSING BOT
}

\author{
Rumaisa Shameem \\ Student, Computer Science Engineering \\ Ssm College of Engineering And \\ Technology,Kashmir,India
}

\author{
Sunaila Mushtaq \\ Student, Computer Science Engineering \\ Ssm College of Engineering And \\ Technology,Kashmir,India,
}

\author{
Mrs Yasmeen \\ Associate Professor \\ Computer Science Engineering \\ Ssm College of Engineering And Technology, Kashmir, India,
}

\begin{abstract}
Humans need to get the things done more easily with the change in day to day technology. So when we use such a technology that has been controlled by some graphical things, it become more easier to work. Weather sensing Bot is actually a wireless Bot that can be controlled by GUI (graphical user interface) and MATLAB. One can built it on Arduino and MATLAB where has interfaced both the software. Communication technique called USART (Universal Synchronous Asynchronous Receiver and Transmitter) can be used for sending data serially via NRF receiver and transmitter. With the help of this Bot various gases, temperature and humidity can be detected by the use of sensors and the information is displayed onto our serial monitor in Arduino.
\end{abstract}

Keywords: MATLAB, Arduino, NRF ( Nordiac Radio Frequency), TTL ( Transistor-Transistor Logic) ,Sensors ( DHT , MQ sensor , Ultrasonic)

\section{INTRODUCTION}

MATLAB [1] has been one of the most extensively used software For most of the field of researches and engineering,. Most of the weather sensing robots till now are made, using its control over buttons or axis based hardware remote control. MATLAB can be used to make a GUI based remote, which may control locomotion of Bot. A bidirectional NRF can be used to send and receive data at both the ends. One can also monitor on computer screen as well as can control the same.

GUI based weather sensing Bot can be made using Aduino IDE[3], and MATLAB[1].GUI. It can employe a virtual remote using graphical user interface in its GUI toolbox. The data which will be sending from GUI will be serially transmitted via TTL. The Data has a baud rate of 9600 that will be sending serially. Also TTL(Transistor-Transistor Logic) can be which can be connected to a laptop and can send our instructions to the Arduino Microcontroller. We can also use a temperature /humidity sensor and MQ6 sensor that detect the temperature/humidity and various gases and result can be displayed on the serial monitor of Arduino with a click of a button that can be made in MATLAB .Also an obstacle detection mechanism can be included that have been employed using Ultrasonic sensor[2].

\section{MICROCONTROLLERS AND MICROPROCESSORS}

All of the components needed for a controller were built onto one chip, as a process of miniaturization continued. Thus a one chip computer, or microcontroller[3], was born.
Example of 8-bit microcontrollers is Intel 8051 family and Motorola family.

The word 'Microprocessor' was actually introduced by INTEL Corporation, and they used this term to describe a newly designed calculator-like integrated-circuit. A CPU built into a single VLSI chip is called microprocessor. It contains arithmetic and logic unit(ALU), Instruction decode and control unit, Instruction register, program counter (pc), clock circuit(internal or external), reset circuit (internal or external) and registers. Example of 8-bit Microprocessors is Intel 8085 and 16-bit Intel 8086/8088.

\subsection{Arduino}

Arduino microcontroller is single board computer. These types of systems provide a sets of digital and analog $\mathrm{I} / \mathrm{O}$ pins that can be interfaced to various expansion boards and other circuits. Thus microcontrollers can be programmed, when Arduino project provides an integrated development environment (IDE) that is based on the processing project, which includes support for the $\mathrm{C}$ and $\mathrm{C}++$ programming languages.

Arduino is open-source because its hardware is reasonably priced and development software is free. Arduino project was basically started in Italy for the development low cost hardware for interaction design. One can write programs and create interface circuits to read switches and other sensors with Arduino board, and to control motors and lights with very little effort. Fig 1 shows a simple diagram of Arduino Uno.

Arduino Software: software programs, called sketches, are created using the Arduino IDE.

Arduino Hardware: An Arduino board historically consists of an Atmel 8, 16 or 32 AVR microcontroller. 
Types of Arduino

- Arduino Uno

- Arduino Leonardo

- Arduino LilyPad

- Arduino Mega

- $\quad$ Arduino Mini Pro

- Arduino BT

\section{MATLAB}

MATLAB can be used to make a GUI. It is one of the most widely used language for many engineering applications. One can made graphical user interface using its GUI toolbox. Data which will be sending from GUI will be serially transmitted via TTL that has a baud rate of 9600.TTL which will be connected to our laptop and will be sending instructions to the Arduino microcontroller [4].

\section{DETAILED DESCRIPTION OF HARDWARE USED}

FIG 2 shows the basic block diagram. The component used are shown in Table 1.

Arduino Nano: It is an open source display place used for building electronics projects. It contains both a physical programmable circuit board and a piece of software, or IDE to upload code to the physical board.

Power: Arduino Nano can be powered via the Mini-B connections, 6-20 unregulated external power supply.

Memory: The Nano has a flash memory of $16 \mathrm{~KB}$ or $32 \mathrm{~KB}$ of which $2 \mathrm{~KB}$ is used by bootloader. It has SRAM of $1 \mathrm{~KB}$ Or $2 \mathrm{~KB}$.It also has EEPROM of 512 bytes or $1 \mathrm{~KB}$ (ATmega).

Input and Output: Nano has 14 digital pins which can be used as input or output using , pinMode(), digitalWrite() and digitalRead() functions. Each of these operate at 5 volts and each can receive or provide a maximum of $40 \mathrm{~mA}$ with internal pull-up resistor of 20-50 kOhms.

Communication: If we want to communicate with a computer, another Arduino, or other microcontrollers then Arduino Nano provides us that facility.

Automatic Software reset: We can reset in Arduino Nano by software running on a connected computer.

Transistor-Transistor logic(TTL):It is used for serial transmission and is built from bipolar junction transition and resistors. Because both logic gating functions and amplifying function are done by transistors that is why it is called transistors-transistor logic.

Motor: Motors are used to convert direct current electrical power into mechanical power.

Voltage Regulator: A voltage regulator is an electrical regulator that is designed to maintain a constant voltage level.

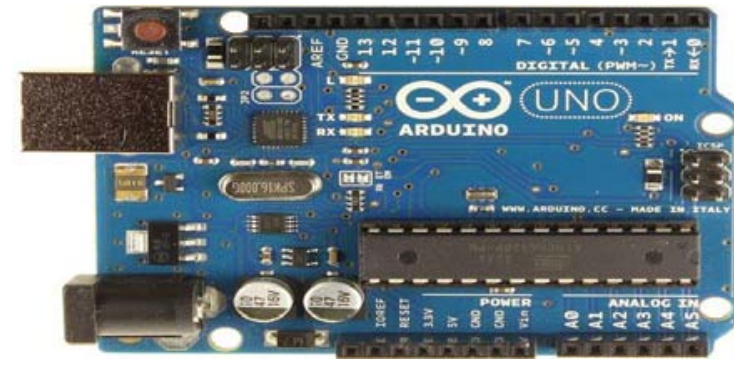

“FIG 1”ARDUINO UNO
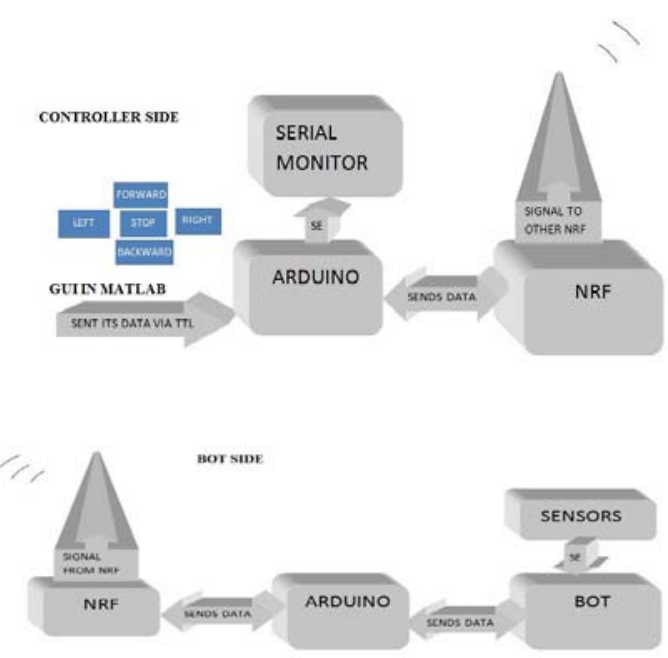

“FIG 2” Block Diagram

TABLE 1. COMPONENTS

\begin{tabular}{|l|l|l|}
\hline Component Used & Quantity & Use \\
\hline Arduino & Two & Microcontroller \\
\hline NRF & Two & Receiver \& Transmitter \\
\hline TTL & One & Serial Communication \\
\hline MQ6 Sensors & One & Gas Detection \\
\hline $\begin{array}{l}\text { Humidity And } \\
\text { Temperature Sensor }\end{array}$ & One & $\begin{array}{l}\text { To Find Humidity Of } \\
\text { The Surronding }\end{array}$ \\
\hline LiPo Battery & One & $\begin{array}{l}\text { Power Supply } \\
\text { To Bot }\end{array}$ \\
\hline Ultrasonic Sensor & One & Obstacle Detection \\
\hline
\end{tabular}

Diode: Diode has two terminals called anode and cathode that means it is a two terminal semiconductor device that exhibits a non linear current-voltage characteristics.

MQ6:MQ6 is used for gas detection. The sensitive material of MQ6 is $\mathrm{SnO} 2$.

Ultrasonic Sensor: It is used for hurdle/obstacle detection.

DHT:It is used to find humidity and temperature of surrounding.

LIPO (Lithium-ion Polymer):Provides power supply to the Bot.

NRF (Nordiac Radio Frequency):In this project we use a bidirectional NRF which sends and receives data at both ends. It acts as a transceiver. 
L293D: L293D is a motor driver means it is a dual H-bridge motor driver integrated circuit(IC).FIG 3 shows pin diagram of L293D.Table 2 gives the pin description of L293D.

\section{ADVANTAGES}

- GUI based weather sensing Bot can be used where a human can't be reached. It sends and receives the data collected from those situations to the other end.

$>$ Because of this Bot, the risk to human lives can be reduced.

$>$ This Bot make the things more and more easy.

$>$ It provides a capability to interact with those surroundings such as volcanic eruptions etc.

\section{CONCLUSION}

GUI based weather sensing Bot can discuss all the mechanical and the hardware aspects as well as the software aspects. This Bot can be controlled in variuos ways with the help of graphical user interface whose programming can be done using microcontroller arduino.The Bot can move forward, backward, left and right using cursor key.Also with the help of sensors,the Bot is able to know various environment conditions. Thus attempts to mix up the hardware and software in a delightful way.

\section{FUTURE SCOPE}

The future of this GUI based weather sensing Bot may include possibly the: wide range cameras , infrared cameras, higher MQ sensors that detect wide range of gaseous compounds.One can also incorporate in this GPS that will give speed and location of Bot.Others may be addition of GSM module, powerful and efficient power supply.

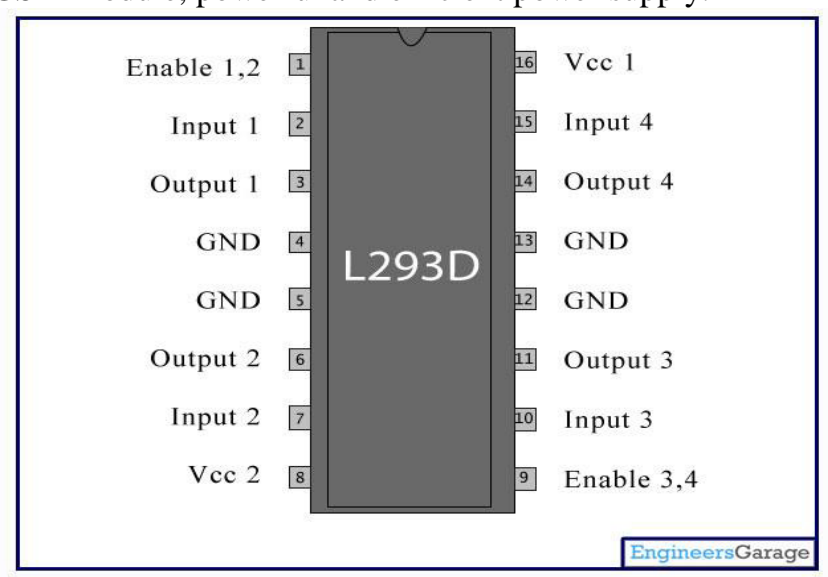

“FIG 3”Pin Diagram
TABLE 2. Pin Description of L293D

\begin{tabular}{|l|l|l|}
\hline Pin No & Function & Name \\
\hline 1 & $\begin{array}{l}\text { Enable pin for Motor 1; } \\
\text { active high }\end{array}$ & Enable 1,2 \\
\hline 2 & Input 1 for Motor 1 & Input 1 \\
\hline 3 & Output 1 for Motor 1 & Output 1 \\
\hline 4 & Ground (0V) & Ground \\
\hline 5 & Ground (0V) & Ground \\
\hline 6 & Output 2 for Motor 1 & Output 2 \\
\hline 7 & Input 2 for Motor 1 & Input 2 \\
\hline 8 & $\begin{array}{l}\text { Supply voltage for Motors; 9- } \\
\text { 12V (up to 36V) }\end{array}$ & Vcc 2 \\
\hline 9 & $\begin{array}{l}\text { Enable pin for Motor 2; } \\
\text { active high }\end{array}$ & Enable 3,4 \\
\hline 10 & Input 1 for Motor 1 & Input 3 \\
\hline 11 & Output 1 for Motor 1 & Output 3 \\
\hline 12 & Ground (0V) & Ground \\
\hline 13 & Ground (0V) & Ground \\
\hline 14 & Output 2 for Motor 1 & Output 4 \\
\hline 15 & Input2 for Motor 1 & Input 4 \\
\hline 16 & $\begin{array}{l}\text { Supply voltage; 5V (up to } \\
\text { 36V) }\end{array}$ & Vcc 1 \\
\hline
\end{tabular}

\section{REFERENCES}

[1] Esther Resendiz, Member, IEEE, John M, Hart, and Narendra Ahuja, Fellow, IEEE” Automated Visual Inspection of Railroad Tracks" IEEE transaction on intelligent transportation systems, vol 14, no.2, June 2013

[2] Isabelle Tang and To by P.Breckon, "Automatic Road environment classification" IEEE transaction on intelligent transportation system, vol 12, no.2, June 2011.

[3] Awati J.S, Patil V.S, “Automatic Irrigation Control by using wireless sensor networks”, Journal of Exclusive Management Science- June 2012-Vol 1 Issue 6.

[4] "Arduino Introduction" arduino.cc. "The Making Of Arduino”.IEEE Spectrum , David Kushner (2011-10-26).

\section{BIOGRAPHIES}

Rumaisa Shameem, is pursuing B.E Degree from SSM College of Engineering \& Technology in Computer Science Engineering, University of Kashmir, J\&K, India. Area of interest is artificial intelligence, MATLAB, robotics.

Sunaila Mushtaq, is pursuing B.E Degree from SSM College of Engineering \& Technology in Computer Science Engineering, University of Kashmir, J\&K, India. Area of interest is artificial intelligence, MATLAB, robotics.

Mrs Yasmeen, is the Associate Professor at SSM college and Engineering and Technology in Department of Computer Science Engineering, University of Kashmir, J\&K, India fields of interest are Computer graphics , data mining and networking. 